\title{
GESTIÓN DE LA LEGITIMIDAD EN LAS PRÁCTICAS DE CALIDAD DE LOS HOTELES
}

\section{Sánchez-Fernández, María Dolores ${ }^{1}$ \\ Universidade da Coruña \\ msanchezf@udc.es}

Material original autorizado para su primera publicación en la revista académica

REDMARKA. Revista Digital de Marketing Aplicado.

https://doi.org/10.17979/redma.2014.01.013.4868

Recibido: 1 Julio 2014

Aceptado 24 Noviembre 2014

\section{Resumen}

El propósito de esta investigación es estudiar la relación entre los principales elementos que conforman las prácticas de calidad y la legitimidad moral en el contexto institucional de los hoteles de tres, cuatro y cinco estrellas ubicados en la Región Norte de Portugal. El marco teórico en el que apoyamos nuestro estudio es la Teoría Institucional, la cual se cimienta en tres pilares esenciales regulativo, normativo y mimético (Scott, 1995). El eje principal de esta teoría es la legitimidad, factor clave para la organización (Meyer y Rowan, 1997; Schuman, 1995; Deephouse, 1996; Egels-Zandén y Wahlqvist, 2007, entre otros). Reconocen Ahlstron y Bruton (2001); Chen, Grifth y Hu (2006) y Diez, Blanco y Prado (2010) que la legitimidad es de vital importancia ya que su deterioro o pérdida puede llevar a la empresa al fracaso.

1 Doctora en Competitividad, Innovación y Desarrollo: Análisis Económica y Empresarial por la Universidad de A Coruña. Desde 2009 personal docente e investigador de la Universidad de A Coruña, pertenece al Departamento de Análisis Económico y ADE, área de organización de empresas. Miembro del Comité Técnico de Asociación para el Desarrollo de la Responsabilidad Empresarial y Social. Miembro colaborador del CICS, Universidade do Minho. Portugal. Miembro colaborador del grupo de investigación GEIDETUR de la Universidad de Huelva, España y Miembro investigador del grupo GREFIN de la Universidad de A Coruña, España.

REDMARKA UIMA-Universidad de A Coruña - CIECID

Año VII, Número 13, (2014), v I pp. 19-42

http://www.redmarka.org/

ISSN 1852-2300 
En el presente trabajo se llevó a cabo una investigación cuantitativa en el que se aplicó la técnica de análisis factorial, con el fin de comprobar la fiabilidad de las escala de medidas propuestas en nuestro objeto de estudio. Mediante el análisis estadístico descriptivo estudiamos entorno institucional, las practicas de calidad y la legitimidad moral de los hoteles objeto de investigación.

La evidencia empírica de este trabajo nos muestra que los principales grupos de interés en la legitimidad moral de la organización son los empleados y los clientes en la implantación de prácticas de calidad. Ello conlleva a que la empresa oriente sus estrategias de legitimidad moral con el fin de gestionarla adecuadamente hacia estos stakeholders. Finalmente resaltar que los resultados del presente estudio son importantes para los gestores de las organizaciones, especial relevancia adquieren los tomadores de decisiones en las áreas de gestión estratégica, marketing y comunicación social.

Palabras clave: legitimidad, prácticas de calidad, hoteles.

\section{Management practices legitimacy of hotel quality}

\section{Abstract}

The purpose of this research is to study the relationship between the main elements of quality practices and moral legitimacy in the institutional context of the hotels of three, four and five star located in the Northern Region of Portugal. The theoretical framework in which we support our study is the Institutional Theory, which is founded on three pillars regulative, normative and mimetic (Scott, 1995). The main focus of this theory is legitimacy, a key factor for the organization (Meyer and Rowan, 1997; Schuman, 1995; Deephouse, 1996; Egels-Zandén and Wahlqvist, 2007, among others). Ahlstron and Bruton (2001); Chen, Grifth and Hu (2006) and Diez, Blanco and Prado (2010) recognize that legitimacy is vital because its deterioration or loss can take the business to fail. In this paper we carried out a quantitative research on the technique of factor analysis was applied to test the reliability of the scale of proposed measures our object of study. We study the institutional environment, quality practices and moral legitimacy of the hotels (we use the descriptive statistical analysis).

\section{REDMARKA UIMA-Universidad de A Coruña - CIECID}


The empirical evidence from this study shows that the main stakeholders in the moral legitimacy of the organization are employees and customers in the implementation of quality practices. This implies that the company focuses its moral legitimacy strategies to properly manage towards these stakeholders. Finally note that the results of this study are important for managers of organizations, acquire special relevance decision makers in the areas of strategic management, marketing and media.

Key words: legitimacy, quality practices, hotels.

\section{Introducción}

En este trabajo analizamos la relación entre los principales elementos que conforman las prácticas de calidad y la legitimidad moral en el contexto institucional de los hoteles de tres, cuatro y cinco estrellas ubicados en la Región Norte de Portugal, apoyándonos los fundamentos de la Teoría Institucional

Esta teoría se cimienta en tres pilares esenciales, el regulativo, el normativo y el cognitivo (Scott, 1995), que dan lugar a las presiones coercitivas, normativas y miméticas emanadas de las instituciones (DiMaggio y Powell, 1991). Estos pilares disponen de un énfasis diferente y derivan en distintas implicaciones. Se identifica la legitimidad como un factor clave para la organización (Meyer y Rowan, 1997; Schuman, 1995; Deephouse, 1996; Egels-Zandén y Wahlqvist, 2007, entre otros) pudiendo adoptar diversas estrategias: ganar, mantener o recuperar legitimidad en la organización (Suchman, 1995). Además de las estrategias existen grupos con capacidad de influir en el ámbito de la legitimidad.

Finalmente, cabe destacar que en el presente trabajo de investigación presentamos una orientación novedosa en la toma de decisiones estratégicas relacionadas con la legitimidad y la adopción de prácticas de calidad por parte de las empresas. La originalidad de este trabajo deriva del conjunto de los principales aportes de esta investigación basados en el marco teórico (Teoría Institucional y calidad), el objeto de estudio y el área geográfica (hoteles de

REDMARKA UIMA-Universidad de A Coruña - CIECID

Año VII, Número 13, (2014), v I pp. 19-42

http://www.redmarka.org/

ISSN 1852-2300 
tres, cuatro y cinco estrellas ubicados en la región Norte de Portugal), el contexto (crisis económica), el ámbito de estudio (esfera privada) y el tipo de organizaciones (con ánimo de lucro).

Este capítulo está dividido a cuatro partes. En la primera sección se realiza una revisión de literatura de la Teoría Institucional, la legitimidad y las prácticas de calidad. Seguidamente se presenta la metodología utilizada y el modelo de investigación. En la cuarta sección se muestran los resultados y su discusión, y seguidamente las conclusiones. La parte final del estudio contiene las referencias bibliográficas.

\section{Revisión de la literatura}

\subsection{La Teoría Institucional}

Los tres pilares institucionales identificados por DiMaggio y Powell (1991) son el normativo, coercitivo y cognitivo. Actúan en este contexto institucional tres mecanismos coercitivo, normativo y mimético (Scott, 1995). El pilar coercitivo se desarrolla a través de los requerimientos legales. Las empresas que implementan estas prácticas adaptándose a las normas generalizadas para conseguir legitimidad social atienden a las presiones del pilar normativo. Cuando la corporación se siente influenciada por la tendencia de imitar las practicas de otras organizaciones que consideran excelentes y que tienen alta legitimidad social está actuando bajo el pilar mimético. La base fundamental de la Teoría Institucional es la legitimidad. Apoyándose en esta teoría Sila (2007) señala que las compañías crean estructuras para parecer legítimas ante sus partes interesadas. Identifican en sus investigaciones Baum y Oliver (1991); Suchman (1995); Deephouse (1996) y Castelló y Lozano, 2011 que la legitimidad es fundamental para las organizaciones, esta les permite sobrevivir y también afecta a su crecimiento. Por lo tanto, las compañías deben tener en consideración para su desarrollo estratégico la adecuada adaptación al entorno institucional, con el fin de alcanzar o mantener legitimidad social. Todo ello conlleva a que la dirección de las empresas gestione la legitimidad de sus organizaciones (Castelló y Lozano, 2011).

REDMARKA UIMA-Universidad de A Coruña - CIECID 
Por todo ello, la Teoría Institucional constituye un marco adecuado para estudiar el contexto institucional de las organizaciones y sus efectos en la legitimidad cuando se implementan prácticas de calidad.

\subsection{La legitimidad}

Suchman (1995) identifica legitimidad moral en una organización cuando esta actúa en concordancia de lo esperado dentro del sistema social. Este autor manifiesta que es un tipo de legitimidad que es difícil de lograr y de manipular. Castelló y Lozano (2011) reconocen que los grupos de interés pueden atribuir este tipo de legitimidad a las organizaciones, cuando estos perciben que obtendrán beneficio de las actividades de la corporación. Por lo que identificar los grupos de interés más importantes para la organización es de vital importancia con el fin de gestionar adecuadamente sus estrategias.

Para las empresas la legitimidad es un factor de vital importancia (Meyer y Rowan, 1977; DiMaggio y Powell, 1983; Singh, Tucker y House, 1986; Fombrun y Shanley, 1990; Scott, 1995; Suchman, 1995; Deephouse, 1996; Vanhonacker, 2000; Ahlstrorn y Bruton, 2001; Chen, Griffith y Hu, 2006; Díez, Blanco y Prado, 2010 y Egels-Zandén y Wahlqvist, 2007) y constituye el eje central de la Teoría Institucional. El deterioro o carencia de la legitimidad puede llevar a una empresa al fracaso (Ahlstrorn y Bruton, 2001; Chen, Griffith y Hu, 2006; Díez, Blanco y Prado, 2010). Por lo tanto, es clave para la organización disponer de un nivel adecuado de legitimidad (Egels-Zandén y Wahlqvist, 2007), representa un factor que regula el éxito o fracaso de la organización (Díez, Blanco y Prado, 2010), ya que esta puede regular el acceso a recursos que le son necesarios a la organización. Estos mismos autores indican que se pueden implementar en la organización determinadas prácticas que permiten gestionar la legitimidad de forma estratégica.

Suchman (1995) identifica tres tácticas que puedan seguir las empresas en función de sus necesidades: ganar, mantener o recuperar la legitimidad. La estrategia de recuperación se fundamenta en respuestas de tipo reactivo, es decir, las empresas reaccionan ante las necesidades de las creencias del sistema social. La estrategia de mantenimiento trata de conservar la legitimidad

REDMARKA UIMA-Universidad de A Coruña - CIECID

Año VII, Número 13, (2014), v I pp. 19-42

http://www.redmarka.org/

ISSN 1852-2300 
lograda pese a los cambios que se puedan producir. Y la estrategia basada en ganar legitimidad permite, mediante la aplicación de determinadas acciones, alcanzar mayor grado de legitimidad a la organización.

\subsection{Prácticas de Calidad}

Con referencia a la calidad, existen muchas definiciones entorno a esta temática, desde las que se conceptualizan en base a la percepción del proveedor del servicio, las que se apoyan en el producto así como las que se orientan al usuario. Por lo tanto en este trabajo se ha adoptado en establecer el criterio de recoger la definición de la calidad más adaptada al objeto de investigación (los hoteles) y el informante (la perspectiva de quien establece la estrategia de la calidad a seguir en la organización, el ápice estratégico de la organización). Además la elección de esta definición se apoya en la tendencia a la universalidad de la gestión de la calidad de las empresas, en este caso es la norma UNE-EN ISO 9000:2008. Se trata de una norma internacional adoptada para gestionar la calidad en el entorno empresarial y ampliamente implementada a nivel internacional, tanto en organizaciones públicas como privadas. Se trata de estándares que hacen referencia a la normalización de aspectos muy diversos de la actividad empresarial. Señala la norma UNE-EN ISO 9000:2008 que, la dirección de la empresa adquiere un papel relevante en la implementación de prácticas de calidad. A través de su liderazgo y sus acciones, la alta dirección puede crear un ambiente en el que el personal se encuentre completamente involucrado y en el cual un sistema de gestión de la calidad puede operar eficazmente. Por todo ello, se cree relevante definir la calidad desde la perspectiva de la dirección de la empresa. Consecuentemente, nos apoyamos en la definición aportada por la (ISO, 2008), entendemos por calidad el grado en el que un conjunto de rasgos diferenciadores bien inherentes al producto, al proceso o sistema cumple con las necesidades o expectativas establecidas de manera implícita u obligatoria a sus partes interesadas.

Varios autores se han basado en Teoría Institucional para desarrollar su investigación, orientada principalmente hacia el medio ambiente (Rivera, 2004;

REDMARKA UIMA-Universidad de A Coruña - CIECID

Año VII, Número 13, (2014), v I pp. 19-42

http://www.redmarka.org/

ISSN 1852-2300 
Vargas-Sánchez y Riquel-Ligero, 2012; Ganapathy, Natarajan Gunasekaran y Subramanian, 2014). En torno a la responsabilidad y la sostenibilidad han desarrollado sus investigaciones Sánchez-Fernández, Vargas-Sánchez y Remoaldo (2014) y Glover, Champion, Daniels and Dainty (2014). En materia de calidad, tomando como marco la Teoría Institucional se encuentra la investigación de Sila (2007), dentro de la principal literatura académica. Este trabajo está enfocado a investigar el contexto institucional y la legitimidad moral que se pueden presentar en la aplicación de las prácticas de calidad. Se extrae de la revisión de la principal literatura académica que no hay muchos estudios empíricos que examinaron el efecto de las medidas de las prácticas de calidad en el marco de la Teoría Institucional. En el siguiente apartado presentamos la metodología.

\section{Metodología}

Este trabajo se ha basado en un estudio cuantitativo, recogiendo la información por medio de un cuestionario dirigido a los directores y directoras de los hoteles del área geográfica objeto de estudio (Región Norte de Portugal). El cuestionario está estructurado en cinco bloques, los dos primeros recogen las principales características que definen a los hoteles y la información sociodemográfica de los directivos de estas entidades. Las dos primeras secciones se construyen en base a informes y documentos elaborados por los principales organismos oficiales de estadística (Instituto de Estadística de Portugal y Cuenta Satélite de Turismo de Portugal). Las tres secciones siguientes corresponden a diferentes escalas utilizadas en diferentes tipos de empresas en otros estudios (Deephouse 1996; Fernández 2001; Kostova e Roth 2002; Llamas 2005; Riquel 2010; Gallardo-Vazquez y Sánchez-Hernández, 2012; Vargas-Sánchez y Riquel-Ligero 2012; Llamas-Sánchez et al., 2013, Gallardo et al. 2013) que miden el contexto institucional, las prácticas de calidad y la legitimidad. En el presente trabajo se validan las escalas en su conjunto y para el objeto de estudio en cuestión en pruebas preliminares, con el fin de minimizar el sesgo que se pudiera producir (Sila, 2014).

REDMARKA UIMA-Universidad de A Coruña - CIECID 
La escala contexto institucional se adapta de los estudios de Kostova y Roth (2002), Vargas-Sánchez y Riquel-Ligero (2012) y Sánchez-Llamas et al. (2013, la cual está compuesta por tres factores, los cuales los conforman las tres presiones institucionales (DiMaggio y Powell, 1991). La escala de prácticas de calidad se ha adaptado del trabajo elaborado por Gallardo-Vázquez y SánchezHernández, 2012 y Gallardo et al. (2013). La escala legitimidad se ha recogido de los estudios de Deephouse (1996), Vargas-Sánchez y Riquel-Ligero (2012) y Sánchez-Llamas et al. (2013).

Entre los ítems que forman parte del contexto institucional está la presión normativa compuesta por 3 elementos: obligación moral $(\mathrm{Cl} 5)$, congruencia con valores del entorno (Cl6) y normas sociales ( $\mathrm{Cl} 7)$; la presión coercitiva está compuesta por 4 ítems: conocimiento de las leyes (CI1), cumplimiento de las leyes $(\mathrm{Cl} 2)$, organismos reguladores $(\mathrm{Cl} 3)$ y existencia de acuerdos $(\mathrm{Cl} 4)$, y la presión mimética está compuesta por otros cuatro variables: conocimiento de experiencias (Cl8), modelos a seguir (CI9), imitación de practicas (Cl10) y conocimiento de experiencias exitosas ( $\mathrm{Cl} 11)$.

Estas tres presiones las relacionamos con las practicas de calidad, factor compuesto por 5 elementos: productos y servicios de calidad (Q1); productos y servicios que cumplen un estándar (Q2); mejores niveles de precios (Q3); información precisa de productos y servicios (Q4) y derechos de los consumidores (Q5).

Y las prácticas de calidad las relacionamos con la legitimidad moral. La legitimidad moral se compone de 9 elementos, conformando la legitimidad de los diferentes grupos de interés: administración pública (LM1); empleados (LM2); ciudadanos (LM3); medios de comunicación (LM4); clientes (LM5); proveedores (LM6); asociaciones (LM7); sector empresarial (LM8) y relaciones con otros grupos de presión (LM9).

Una vez elaborado el cuestionario, este fue revisado por tres especialistas en las áreas de gestión, la geografía y turismo. Después de esta revisión se añadieron las sugerencias propuestas por los revisores al pre-test, el cual se llevó a cabo durante el mes de abril de 2012. Durante este mes se recogieron

REDMARKA UIMA-Universidad de A Coruña - CIECID 
cinco cuestionarios procedentes de cinco gerentes de hotel de la Región Norte de Portugal. Finalmente la encuesta se envió a la población universo de estudio desde el mes de mayo de 2012 hasta agosto de este mismo año, recogiendo la información de la muestra mediante diferentes formas de contacto (correo electrónico, formulario web, fax, contacto telefónico y contacto presencial en el hotel).

El tamaño total de la muestra se compone de 44 hoteles. Se obtuvo una tasa de respuesta del $29,9 \%$, con un error del $5 \%$ en el que $p=q=0,5$. Consideramos estos porcentajes como adecuados, el nivel más exigente se estableció en base a los estudios desarrollados por Vargas-Sánchez y Riquel-Ligero (2012), los cuales obtuvieron una tasa de respuesta del 33\%. Otras tasas de respuesta más bajas fueron descartadas, tales como los obtenidas por Llamas-Sánchez et al. (2013), Gallardo-Vázquez y Sánchez-Hernández, 2012 y Gallardo et al. (2013), con porcentajes que van del 21\% al 14\%. Para reforzar aún más la afirmación anterior hemos sometido a la variable número de estrellas de los hoteles a un test de medias con el software SPSS, para ello hemos contrastado y calculado el intervalo de confianza con respecto la media de la categoría de la población que se sitúa en 3,59 estrellas por hotel, la desviación típica obtiene un valor de 0,680 y un error típico de la media muestra un resultado de 0,103. Además también hemos realizado la prueba para una muestra según la categoría del hotel, siendo el valor de la muestra de 3,59. Con lo que podemos concluir que bien por el intervalo de confianza, bien por el valor del estadístico, debemos aceptar la hipótesis nula y considerar que el número medio de estrellas de los hoteles ubicados en la región Norte de Portugal es 3,59, existiendo una ligera diferencia con la media muestral.

El modelo de investigación propuesto (ver figura 1) nos permitirá responder a las siguientes preguntas:

¿Se corrobora que el entorno institucional de nuestro objeto de estudio está compuesto por las tres presiones institucionales identificadas por DiMaggio y Powell (1991)?

REDMARKA UIMA-Universidad de A Coruña - CIECID

Año VII, Número 13, (2014), v I pp. 19-42

http://www.redmarka.org/

ISSN 1852-2300 
¿Cuáles son los elementos que ejercen mayor influencia en el contexto institucional de nuestro objeto de estudio?

¿Cuáles son los stakeholders más relevantes en la legitimidad moral?

¿Cuáles son las variables que ejercen mayor influencia en las prácticas de calidad de nuestro objeto de estudio?

Figura 1- Modelo de investigación propuesto

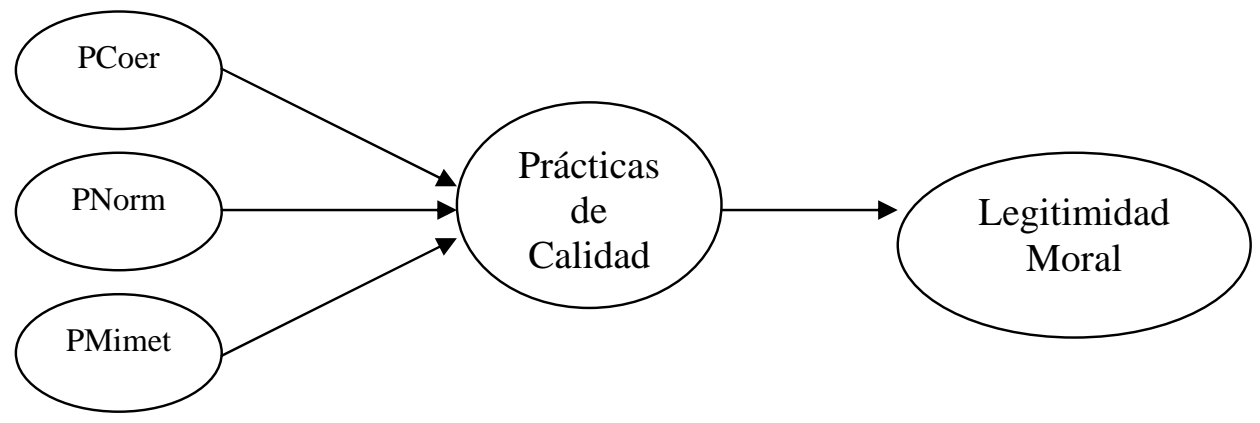

Fuente: Elaboración propia

Para dar respuesta a las preguntas planteadas sometemos las variables a pruebas estadísticas descriptivas y de análisis factorial (Bartlett 1951; Kaiser 1970; Nunnally 1978; Wubneh 1987; Verdu 2002; Aymerich and Meseguer 2004; Perez 2005; Wang 2005) mediante la aplicación del SPSS (versión 18.0). Los criterios básicos en los que nos apoyamos para aceptar o descartar los factores y las variables que los conforman están reflejados en la Tabla 1. Obteniendo así los resultados estadísticos y los conjuntos de variables observables que presentaban un valor añadido a factores que conforman nuestro modelo. La técnica de análisis factorial permitió reducir la información contenida en el conjunto original de variables a un conjunto más pequeño de ítems con una pérdida mínima de información.

Tabla 1: Criterios de las pruebas del análisis factorial

\begin{tabular}{|l|l|}
\hline \multicolumn{1}{|c|}{ Pruebas } & \multicolumn{1}{c|}{ Criterios } \\
\hline Matriz de correlaciones & Existe un 60\% de correlaciones $>0,30$ \\
\hline Valor determinante & Muy bajo \\
\hline KMO & $>0,5$ (Verdu, 2002; Pérez, 2005) \\
\hline $\begin{array}{l}\text { Prueba esfericidad de } \\
\text { Bartlett }\end{array}$ & $\begin{array}{l}\text { Nivel de significación (Sig) }<0,05 \text { (Bartlett 1950; } \\
1951)\end{array}$ \\
\hline
\end{tabular}

REDMARKA UIMA-Universidad de A Coruña - CIECID Año VII, Número 13, (2014), v I pp. 19-42 http://www.redmarka.org/ 


\begin{tabular}{|l|l|}
\hline Alfa Cronbach & Escala fiable $>0,7$ (Nunnally, 1978) \\
\hline Comunalidades & Tendencia a 1 \\
\hline Varianza total explicada & $\begin{array}{l}\text { Rango mínimo (60-80\%) (Wubneh, 1987; Wang, } \\
\text { 2005) }\end{array}$ \\
\hline $\begin{array}{l}\text { Matriz de componentes } \\
\text { rotados }\end{array}$ & $\begin{array}{l}\text { Especificación número de factores esperado según } \\
\text { cantidad de información restituida }\end{array}$ \\
\hline
\end{tabular}

Fuente: Sánchez-Fernández (2014, p. 162)

Bajo la prueba de la unidimensionalidad de las escalas analizamos las diferentes secciones que nos aportan información a través del análisis factorial. Con carácter previo al análisis factorial estudiamos si la matriz de correlaciones es adecuada para aplicar esta técnica, para ello utilizamos el determinante de la matriz de correlaciones. Realizamos la prueba KMO para contrastar si las correlaciones parciales entre las variables son suficientemente pequeñas. Seguidamente sometemos los datos a la prueba de esfericidad de Bartlett con el fin de contrastar la hipótesis nula, la matriz de correlaciones es una matriz identidad. Utilizamos el Alfa de Cronbach para estudiar la fiabilidad. Posteriormente estudiamos las comunalidades, la varianza explicada y el gráfico de sedimentación. Finalmente analizamos la matriz de componentes principales y, en caso de obtener más de un factor tras la rotación, analizamos la matriz de componentes rotados y el gráfico de componentes en espacio rotado. Para todo ello analizamos las siguientes escalas: contexto institucional, legitimidad moral y prácticas de calidad.

El caso que estudiamos contempla diferentes variables, lo que dificulta su agrupación, ya que queremos diferenciar los distintos tipos de factores enmarcados en nuestro modelo y así analizar sus características particulares. Si pudiéramos agrupar en unos pocos factores todas las variables que nos proporcionan información, estaríamos en mejor disposición de hacer dicha agrupación. De entre estas técnicas factoriales, una de las más conocidas es la de componentes principales. Esta es la técnica factorial en la que nos centraremos y está basada en las correlaciones que existen entre las diferentes variables. La diagonalización de la matriz de correlaciones es el punto clave del

REDMARKA UIMA-Universidad de A Coruña - CIECID

Año VII, Número 13, (2014), v I pp. 19-42

http://www.redmarka.org/

ISSN 1852-2300 
procedimiento de obtención de los componentes principales. En el siguiente epígrafe exponemos los resultados y su discusión.

\section{Resultados y discusión}

Seguidamente presentamos los resultados obtenidos y su discusión, una vez aplicada la metodología detallada en el apartado anterior. Resaltamos en primer lugar las principales características de los hoteles objeto de estudio. La distribución de los hoteles según el área geográfica es de: Porto 50\%, Braga 20\%, Viana do Castelo 12\%, Vila Real 9\%, Viseu 7\% y Bragança 2\%, manteniéndose coherencia en la representación geográfica de los hoteles entre la muestra y el universo de estudio. En la misma línea, muestra coherencia entre la muestra y el universo de estudio la distribución según la categoría del hotel, obteniendo que el $46 \%$ son hoteles de 3 estrellas, $43 \%$ hoteles de 4 estrellas y 11\% hoteles de 5 estrellas. En cuanto ámbito geográfico donde operan los hoteles, el $46 \%$ pertenecen al conjunto de ámbitos local, regional, autonómico y provincial, el $29 \%$ son de ámbito internacional, y el $25 \%$ corresponde al ámbito nacional.

A continuación se exponen las principales características sociodemográficas de la dirección de los hoteles, según el género el 34\% son mujeres ejecutivas y el $66 \%$ restante corresponde a los hombres directivos. Los porcentajes obtenidos en nuestro estudio en cuanto a la representación según el género se consideran adecuados en base a los estudios de Alonso-Almeida (2011) y Alonso-Almeida (2013). Según el grupo de edad, hemos establecido 8 intervalos que representan el rango de edad de los directivos/as de los hoteles. El mayor porcentaje lo representan aquellos comprendidos entre los 41-45 años con un 27,3\%, siguiendo el rango de edad superior e inferior, entre 36-40 años el 18,2\% y 56-50 años el 20,5\%. Con referencia al nivel de educación, el $69 \%$ disponen de estudios superiores universitarios, el 30\% en dos niveles, superior e inferior al de estudios universitarios (el 15\% estudios secundarios y el $15 \%$ corresponden a estudios de nivel de postgraduado, master o

\section{REDMARKA UIMA-Universidad de A Coruña - CIECID}


doctorado), tan sólo el 1\% de la dirección de los hoteles dispone de estudios básicos. De los datos se desprende que el perfil de la dirección de los hoteles objeto de estudio es un varón cuya edad está comprendida entre los 41 y 45 años con estudios universitarios superiores.

En el siguiente apartado estudiaremos los estadísticos descriptivos.

\subsection{Estadísticos descriptivos}

Para dar respuesta a las preguntas inicialmente planteadas, en primer lugar estudiaremos los estadísticos descriptivos del contexto institucional. Mostramos en la Tabla 2 los resultados obtenidos de los estadísticos descriptivos de las escalas propuestas en nuestro estudio, contexto institucional, prácticas de calidad y legitimidad moral.

REDMARKA UIMA-Universidad de A Coruña - CIECID

Año VII, Número 13, (2014), v I pp. 19-42

http://www.redmarka.org/

ISSN 1852-2300 
Tabla 2: Estadísticos descriptivos

\begin{tabular}{|c|c|c|c|c|c|}
\hline Escalas & Ítem & Ref. & Moda & Media & $\begin{array}{c}\text { Desviación } \\
\text { típica }\end{array}$ \\
\hline \multirow{11}{*}{$\begin{array}{l}\text { Contexto } \\
\text { Institucional }\end{array}$} & Conocimiento en Leyes & $\mathrm{Cl} 1$ & 4 & 3,66 & 1,160 \\
\hline & Cumplimiento de Leyes & $\mathrm{Cl} 2$ & 4 & 4,18 & 0,843 \\
\hline & Organismos reguladores & $\mathrm{Cl} 3$ & 4 & 3,52 & 1,151 \\
\hline & Existencia de Acuerdos & $\mathrm{Cl} 4$ & 4 & 3,61 & 1,205 \\
\hline & Obligación Moral & $\mathrm{Cl} 5$ & 4 & 4,14 & 0,852 \\
\hline & $\begin{array}{l}\text { Congruencia con valores del } \\
\text { entorno }\end{array}$ & $\mathrm{Cl} 6$ & 4 & 4,25 & 0,751 \\
\hline & Normas Sociales & $\mathrm{Cl} 7$ & 4 & 4,05 & 0,776 \\
\hline & Conocimiento de experiencias & $\mathrm{Cl} 8$ & 4 & 3,48 & 0,976 \\
\hline & Modelos a seguir & $\mathrm{Cl9}$ & 4 & 3,11 & 0,993 \\
\hline & Imitación prácticas & $\mathrm{Cl} 10$ & 2 & 2,61 & 0,920 \\
\hline & $\begin{array}{l}\text { Conocimiento experiencias } \\
\text { exitosas }\end{array}$ & Cl11 & 3 & 3,20 & 1,025 \\
\hline \multirow{5}{*}{$\begin{array}{l}\text { Prácticas de } \\
\text { calidad }\end{array}$} & $\begin{array}{l}\text { Productos y servicios de } \\
\text { calidad }\end{array}$ & Q1 & 5 & 4,59 & 0,693 \\
\hline & $\begin{array}{l}\text { Productos y servicios cumplen } \\
\text { un estándar }\end{array}$ & Q2 & 5 & 4,66 & 0,608 \\
\hline & Mejores niveles de precios & Q3 & 5 & 4,77 & 0,522 \\
\hline & $\begin{array}{l}\text { Información precisa de } \\
\text { productos y servicios }\end{array}$ & Q4 & 5 & 4,57 & 0,625 \\
\hline & $\begin{array}{l}\text { Derechos de los } \\
\text { consumidores }\end{array}$ & Q5 & 5 & 4,61 & 0,618 \\
\hline \multirow{9}{*}{$\begin{array}{l}\text { Legitimidad } \\
\text { moral }\end{array}$} & Legitimidad Admon. Pública & LM1 & 4 & 3,66 & 0,987 \\
\hline & Legitimidad Empleados & LM2 & 5 & 4,27 & 0,872 \\
\hline & Legitimidad Ciudadanos & LM3 & 3 & 3,57 & 1,108 \\
\hline & $\begin{array}{l}\text { Legitimidad Medios de } \\
\text { Comunicación }\end{array}$ & LM4 & 3 & 3,36 & 0,990 \\
\hline & Legitimidad clientes & LM5 & 5 & 4,14 & 0,905 \\
\hline & Legitimidad Proveedores & LM6 & 4 & 3,91 & 0,884 \\
\hline & Legitimidad Asociaciones & LM7 & 3 & 3,48 & 0,876 \\
\hline & $\begin{array}{l}\text { Legitimidad Sector } \\
\text { Empresarial }\end{array}$ & LM8 & 4 & 3,73 & 0,924 \\
\hline & $\begin{array}{l}\text { Relaciones con otros grupos } \\
\text { de presión }\end{array}$ & LM9 & 5 & 4,84 & 0,428 \\
\hline
\end{tabular}

\section{Fuente: Elaboración propia}

Para responder a la pregunta inicialmente planteada ¿Cuáles son los elementos que ejercen mayor influencia en el contexto institucional de nuestro objeto de estudio? exponemos la discusión de los resultados obtenidos. En el análisis descriptivo de los diferentes ítems referentes al contexto institucional, puede observarse en la tabla 2, en la cual se puede inferir que la presión normativa es el mecanismo institucional que ejerce mayor presión en el contexto institucional de las prácticas de calidad de los hoteles; ya que sus componentes como la congruencia con los valores del entorno obtuvo la media

REDMARKA UIMA-Universidad de A Coruña - CIECID

Año VII, Número 13, (2014), v I pp. 19-42

http://www.redmarka.org/

ISSN 1852-2300 
más alta con un 4.25 y una variabilidad del 0.751, mientras que la obligación moral (con una media de 4.18) y las normas sociales (con una media de 4.05) fueron los elementos que más aplicaban los hoteles. Cabe mencionar, que con una media de 4.18, el cumplimiento de leyes que esta dentro de la presión coercitiva también fue muy valorado. En general, los elementos de la presión mimética fueron los menos valorados.

En cuanto a los estadísticos descriptivos de las prácticas de calidad, nos planteamos la siguiente cuestión ¿Cuáles son las variables que ejercen mayor influencia en las prácticas de calidad de nuestro objeto de estudio? Distinguirse por mantener los mejores niveles de precios en relación a la calidad ofrecida es el elemento con mayor importancia para los hoteles de la Región Norte de Portugal. La menos desarrollada por los hoteles es ofrecer información precisa de productos y servicios.

Finalmente comentar los estadísticos descriptivos de la escala legitimidad moral para dar respuesta a la pregunta ¿Cuáles son los stakeholders más relevantes en la legitimidad moral? Se observa que los hoteles, mantener las relaciones estables con otros grupos de interés (no definidos en nuestro listado), los empleados y los clientes son los elementos a los que más se les otorga legitimidad y apoyo social. Ya que estos tienen los valores de medias mas altos como son 4.84, 4.27 y 4.14 respectivamente, así como variaciones pequeñas y la valoración de importancia más alta (5 puntos en su media=totalmente de acuerdo). Una vez estudiados los estadísticos descriptivos presentamos los resultados obtenidos en el análisis factorial.

\subsection{Análisis Factorial.}

En base a los criterios establecidos, ver Tabla 1, presentamos los resultados obtenidos del análisis factorial. Nuestro objetivo es reducir la información contenida en una serie de variables originales, a una serie más pequeña con la mínima perdida de información. Las variables que inicialmente forman parte de las prácticas de calidad, legitimidad moral y contexto institucional son las contempladas en el anterior epígrafe, metodología. A partir de estas variables iniciales queremos realizar un análisis de componentes principales para

REDMARKA UIMA-Universidad de A Coruña - CIECID 
determinar la adecuada agrupación de variables a través de la obtención de un menor número de factores. Exponemos en la Tabla 3 los resultados de las pruebas a las cuales hemos sometido las diferentes escalas.

REDMARKA UIMA-Universidad de A Coruña - CIECID

Año VII, Número 13, (2014), v I pp. 19-42

http://www.redmarka.org/

ISSN 1852-2300 
Tabla 3: Tabla resumen pruebas de las escalas

\begin{tabular}{|c|c|c|c|c|c|c|c|}
\hline \multirow{2}{*}{\begin{tabular}{|l|} 
\\
$1^{\circ}$ fase \\
\end{tabular}} & \multirow[t]{2}{*}{ Criterios } & \multicolumn{2}{|c|}{$\begin{array}{c}\text { Contexto } \\
\text { Institucional }\end{array}$} & \multicolumn{2}{|c|}{$\begin{array}{c}\text { Legitimida } \\
\mathrm{d}\end{array}$} & \multicolumn{2}{|c|}{ Calidad } \\
\hline & & & & & & & \\
\hline $\begin{array}{l}\text { Matriz de } \\
\text { correlaciones }\end{array}$ & $\begin{array}{lll}\text { Existe un } 60 \% & \text { de } \\
\text { correlaciones }>0,30 & \\
\end{array}$ & $\mathrm{Si}$ & $\checkmark$ & $\mathrm{Si}$ & $\checkmark$ & Si & $\checkmark$ \\
\hline $\begin{array}{l}\text { Valor } \\
\text { determinante }\end{array}$ & Muy bajo & 0,000 & $\checkmark$ & 0,003 & $\checkmark$ & 0,015 & $\checkmark$ \\
\hline $\mathrm{KMO}$ & $\begin{array}{l}>0,5 \text { (Verdu, 2002; Pérez, } \\
2005)\end{array}$ & 0,624 & $\checkmark$ & 0,741 & $\checkmark$ & 0,858 & $\checkmark$ \\
\hline $\begin{array}{l}\text { Prueba } \\
\text { esfericidad de } \\
\text { Bartlett }\end{array}$ & $\begin{array}{l}\text { Nivel de significación (Sig) < } \\
0,05 \text { (Bartlett 1950; 1951) }\end{array}$ & 0,000 & $\checkmark$ & 0,000 & $\checkmark$ & 0,000 & $\checkmark$ \\
\hline Alfa Cronbach & $\begin{array}{l}\text { Escala fiable }>0,7 \text { (Nunnally, } \\
\text { 1978) }\end{array}$ & 0,828 & $\checkmark$ & 0,881 & $\checkmark$ & 0,925 & $\checkmark$ \\
\hline $\begin{array}{l}\text { Comunalidade } \\
\mathrm{s}\end{array}$ & Tendencia a 1 & $\mathrm{Si}$ & $\checkmark$ & $\mathrm{Si}$ & $\checkmark$ & $\mathrm{Si}$ & $\checkmark$ \\
\hline $\begin{array}{l}\text { Varianza total } \\
\text { explicada }\end{array}$ & $\begin{array}{l}\text { Rango mínimo }(60-80 \%) \\
\text { (Wubneh, } 1987 ; \text { Wang, 2005) }\end{array}$ & $82,1 \%$ & $\checkmark$ & $\begin{array}{l}73,8 \\
\%\end{array}$ & $\checkmark$ & $77,5 \%$ & $\checkmark$ \\
\hline $\begin{array}{l}\text { Matriz de } \\
\text { componentes } \\
\text { rotados }\end{array}$ & $\begin{array}{llr} & \text { factor } & \text { (Calidad y } \\
\text { Legitimidad) } & \\
3 \quad \text { factores } & \text { (Contexto } \\
\text { Institucional) } & \\
\end{array}$ & 4 & $x$ & 2 & $x$ & 1 & $\checkmark$ \\
\hline \multicolumn{8}{|l|}{$2^{\circ}$ fase $^{*}$} \\
\hline $\begin{array}{l}\text { Matriz de } \\
\text { correlaciones }\end{array}$ & $\begin{array}{l}\text { Existe un } 60 \% \\
\text { correlaciones }>0,30\end{array}$ & Si & $\checkmark$ & Si & $\bar{v}$ & -- & -- \\
\hline $\begin{array}{l}\text { Valor } \\
\text { determinante }\end{array}$ & Muy bajo & 0,001 & $\checkmark$ & 0,001 & $\checkmark$ & -- & -- \\
\hline $\mathrm{KMO}$ & $\begin{array}{l}>0,5 \text { (Verdu, 2002; Pérez, } \\
2005)\end{array}$ & 0,620 & $\checkmark$ & 0,743 & $\checkmark$ & -- & -- \\
\hline $\begin{array}{l}\text { Prueba } \\
\text { esfericidad de } \\
\text { Bartlett }\end{array}$ & $\begin{array}{l}\text { Nivel de significación (Sig) < } \\
0,05 \text { (Bartlett 1950; 1951) }\end{array}$ & 0,0000 & $\checkmark$ & 0,001 & $\bar{v}$ & -- & -- \\
\hline Alfa Cronbach & $\begin{array}{l}\text { Escala fiable }>0,7 \text { (Nunnally, } \\
\text { 1978) }\end{array}$ & 0,815 & $\checkmark$ & 0,888 & $\checkmark$ & -- & -- \\
\hline $\begin{array}{l}\text { Comunalidade } \\
\mathrm{s}\end{array}$ & Tendencia a 1 & $\mathrm{Si}$ & $\checkmark$ & $\mathrm{Si}$ & $\checkmark$ & -- & -- \\
\hline $\begin{array}{l}\text { Varianza total } \\
\text { explicada }\end{array}$ & $\begin{array}{l}\text { Rango mínimo }(60-80 \%) \\
\text { (Wubneh, } 1987 ; \text { Wang, 2005) }\end{array}$ & $76,1 \%$ & $\checkmark$ & $\begin{array}{l}67,9 \\
\%\end{array}$ & $\checkmark$ & -- & -- \\
\hline $\begin{array}{l}\text { Matriz de } \\
\text { componentes } \\
\text { rotados }\end{array}$ & $\begin{array}{lrr}\text { Legitor } & \text { (Calidad y } \\
\text { Legitimidad) } & \\
3 \quad \text { factores } & \text { (Contexto } \\
\text { Institucional) } & \\
\end{array}$ & 3 & $\checkmark$ & 1 & $\checkmark$ & -- & -- \\
\hline
\end{tabular}

Fuente: Elaboración propia.

En base a los resultados presentados en la Tabla 3, las variables que conforman el contexto institucional se someten a un segundo ajuste, ya que en una primera fase no cumplía los requisitos establecidos. En el caso de la Región Norte de Portugal se extraen cuatro factores, no cumpliéndose así el

REDMARKA UIMA-Universidad de A Coruña - CIECID

Año VII, Número 13, (2014), v I pp. 19-42

http://www.redmarka.org/

ISSN 1852-2300 
requisito teórico por el que deben tener tan solo tres factores; estos componen el contexto institucional en base a los tres pilares institucionales: coercitivo, normativo y mimético (Scott, 1995), derivados de las presiones institucionales identificadas por DiMaggio y Powel (1991). Por lo tanto, eliminamos este cuarto factor en el que tan solo está incluida una variable $\mathrm{Cl} 11$, conocimiento de experiencias exitosas. La decisión se apoya en este hecho y volvemos a someter los datos al análisis factorial para comprobar si cumplen los criterios establecidos en esta segunda fase. El resultado es afirmativo, se cumplen todos los criterios establecidos y obtenemos el número óptimo, tres factores.

Por otra parte, el constructo prácticas de calidad (Q) está constituido por un solo factor en el que se incluyen la totalidad de las variables propuestas inicialmente, cumpliendo, de este modo, todos los criterios previamente establecidos.

En referencia al factor de legitimidad moral, a continuación exponemos las argumentaciones en las que nos basamos para eliminar una de las variables razón por la cual sometemos esta escala a una segunda fase. Para la eliminación de uno de los factores, nos apoyamos en el gráfico de espacio rotado; la variable contenida en el segundo factor no puede formar parte de ninguno de los factores mencionados y se aleja de ellos. Además, apoyamos nuestra decisión en el análisis del gráfico de sedimentación, a partir del primer factor se produce un cambio de concavidad, sostenemos que tan solo deben formarse un factor y no dos. Argumentamos la necesidad de eliminar este segundo factor en base a la facilidad de interpretación de los factores que contribuyen a la formación del factor uno. Además, el factor dos se entremezcla en la interpretación del primer factor, relacionado con los grupos de interés. Por estas razones realizamos un segundo análisis tras la eliminación de la variable LM9, relaciones con otros grupos de presión. Una vez que sometemos las variables seleccionadas a un segundo análisis factorial, se cumplen todos los criterios establecidos.

REDMARKA UIMA-Universidad de A Coruña - CIECID

Año VII, Número 13, (2014), v I pp. 19-42

http://www.redmarka.org/

ISSN 1852-2300 
Seguidamente exponemos los elementos finales que constituyen los constructos que forman parte del contexto institucional, prácticas de calidad y legitimidad moral. En la escala legitimidad moral se conservan todas las variables a excepción de la relación con los grupos de interés; en el caso del factor prácticas de calidad se mantienen todas las inicialmente propuestas y en el contexto institucional, el único que no conserva todas las variables es el factor relacionado con las prácticas miméticas, eliminándose la variable conocimiento de experiencias exitosas, permaneciendo con el mismo conjunto de variables los factores relacionados con prácticas normativas y coercitivas.

Elementos que constituyen los factores que conforman el contexto institucional:

Presión normativa: obligación moral (Cl5), congruencia con valores del entorno $(\mathrm{Cl} 6)$ y normas sociales $(\mathrm{Cl} 7)$;

Presión coercitiva: conocimiento de las leyes ( $\mathrm{Cl} 1)$, cumplimiento de las leyes $(\mathrm{Cl} 2)$, organismos reguladores $(\mathrm{Cl} 3)$ y existencia de acuerdos $(\mathrm{Cl} 4)$;

Presión mimética: conocimiento de experiencias (Cl8), modelos a seguir (CI9) y la imitación de practicas (CI10).

Prácticas de calidad: productos y servicios de calidad (Q1); productos y servicios que cumplen un estándar (Q2); mejores niveles de precios (Q3); información precisa de productos y servicios (Q4) y derechos de los consumidores (Q5).

Legitimidad moral: administración pública (LM1); empleados (LM2); ciudadanos (LM3); medios de comunicación (LM4); clientes (LM5); proveedores (LM6); asociaciones (LM7) y sector empresarial (LM8).

En base a las argumentaciones inicialmente demarcadas en esta investigación, se corrobora que el entorno institucional de nuestro objeto de estudio está compuesto por las tres presiones institucionales identificadas por DiMaggio y Powell (1991): presión coercitiva, normativa y mimética.

\section{Conclusiones}

REDMARKA UIMA-Universidad de A Coruña - CIECID

Año VII, Número 13, (2014), v I pp. 19-42

http://www.redmarka.org/

ISSN 1852-2300 
De los resultados en esta investigación se desprende que la búsqueda de la legitimidad y apoyo social afecta en la implementación de prácticas de calidad. . El principal interés que manifiestan los directivos de los hoteles es el mantener relaciones estables con los stakeholders, especialmente con los clientes y los empleados. En base a la Teoría Institucional las organizaciones crean estructuras para parecer legítimas ante sus partes interesadas (Sila, 2007). En nuestro caso se observa que, el método que se percibe como más legítimo es la implementación de prácticas de calidad entorno al ámbito de las normas orientadas a los clientes y a los empleados.

En el análisis del contexto institucional se desprende que el entorno institucional de nuestro objeto de estudio está compuesto por las tres presiones institucionales identificadas por DiMaggio y Powell (1991): presión coercitiva, normativa y mimética. La presión normativa es el mecanismo institucional que ejerce mayor presión en el contexto institucional; ya que la congruencia con los valores del entorno, la obligación moral y las normas sociales fueron los elementos que mas aplicaban los hoteles. Cabe mencionar, que el cumplimiento de leyes que esta dentro de la presión coercitiva también fue muy valorado. Los elementos de la presión mimética fueron los menos valorados.

En relación a las prácticas de calidad, los hoteles de la Región Norte de Portugal se distinguen principalmente por mantener los mejores niveles de precios en concordancia a la calidad ofrecida. Este es el elemento con mayor importancia en las prácticas de calidad.

Finalmente exponemos las limitaciones de este estudio, a partir de las cuales se proponen futuras líneas de investigación. El tamaño de la muestra es pequeño y no podemos generalizar los resultados a todo el país, sin embargo representan una información útil para las empresas hoteleras y las investigaciones relacionadas con esta temática. En base a esta limitación se recomienda que en futuras investigaciones se trabaje con áreas geográficas de mayor tamaño, todo el país. También sería de gran interés realizar una comparativa entre unidades hoteleras localizadas en diferentes países, con el fin de verificar el comportamiento isomórfico de estas organizaciones. Otra de

REDMARKA UIMA-Universidad de A Coruña - CIECID

Año VII, Número 13, (2014), v I pp. 19-42

http://www.redmarka.org/

ISSN 1852-2300 
las limitaciones de este estudio está relacionada con la recogida de información a través de un solo informante, los directivos de hotel, sesgo que tratamos minimizar con las diferentes pruebas de validez a las que sometimos las escalas. En esta línea, siguiendo las recomendaciones de Kumar, Stern y Anderson (1993) sería relevante recoger información de diferentes stakeholders. Con el fin de delimitar grupos de interés, en base a los resultados de este estudio, los más relevantes para su estudio son los clientes y los empleados.

\section{Referencias Bibliográficas}

Ahlstrom, D. y Bruton, G.D. (2001). Learning from successful local private firms in China: Establishing legitimacy. Academy of Management Executive, 15 (4), $72-83$.

Alonso-Almeida MM. (2011) La dirección y el gobierno de las empresas turísticas desde la perspectiva de género. In Alonso-Almeida, M.M. and Rodriguez-Antón, J.M. (Eds.) Turismo y Género (pp. 49-74). Madrid: Editorial Sintesis.

Alonso-Almeida, M. (2013). Environmental management in tourism: Students' perceptions and managerial practice in restaurants from a gender perspective. Journal of Cleaner Production, 60 (1), 201 - 207.

Aymerich Martínez, J. y Meseguer Artola, A. (2004). Investigación descriptiva: análisis de información. En Meseguer Artola, A. y Vilaseca Requena, J (Coord). Estadística aplicada. Catalunya: Fundació Universitat Oberta de Catalunya.

Bartlett, M.S. (1951). A further note on tests of significance in factor analysis. British Journal of Psychology, 4, 1-2.

Baum, J.A. y Oliver, C. (1991). Institutional linkages and organizational mortality. Administrative Science Quarterly, 36(2), 187 - 219.

Castelló, I. y Lozano, J.M. (2011). Searching for New Forms of Legitimacy Through Corporate Responsibility Rhetoric. Journal of Business Ethics, 100 (1), 11 - 29.

Chen, H.; Griffith, D. y Hu, M. (2006). The influence of liability of foreignness on market entry strategies: An illustration of market entry in China. International Marketing Review, 23 (6), 636 - 649.

Deephouse, D.L. (1996). Does isomorphism legitimate?. Academy of Management Journal, 39 (4), 1024 - 1039.

REDMARKA UIMA-Universidad de A Coruña - CIECID

Año VII, Número 13, (2014), v I pp. 19-42

http://www.redmarka.org/

ISSN 1852-2300 
Díez Martín, F.; Blanco González, A. y Prado Román, C. (2010). Medición de la legitimidad organizativa: El caso de las Sociedades de Garantía Recíproca. Cuadernos de Economía y Dirección de la Empresa, 13 (43), 115 - 143.

DiMaggio, P. J. y Powell, W.W. (1991). The Iron Cage Revisited: Institutional Isomorphism and Collective Rationality in Organization Fields. In W. W. Powell and P. J. DiMaggio (eds.), The New Institutionalism in Organizational Analysis (pp. 63 - 82). Chicago, IL: University of Chicago Press.

Egels-Zandén, N. y Wahlqvist, E. (2007). Post-partnership strategies for Refining corporate responsibility: The business social compliance initiative. Journal of Business Ethics, 70 (2), 175 - 189.

Fernández, M.L. (2001). Un análisis institucional del contexto y su incidencia en el proceso de cambio en la gestión de los recursos humanos. Tres estudios de casos. Tesis Doctoral, Universidad de Cádiz. Facultad de Ciencias Económicas y Empresariales, España.

Fombrun, C. y Shanley, M. (1990). What's in a Name: Reputation Building and Corporate Strategy. Academy of Management Journal, 2, 233-358.

Fornell, C. y Lacker, D. (1981). Evaluating structural equation models with unobservable variables and measurement error: algebra and stadistic. Journal of Marketing Research, 28 (February), 39-50.

Gallardo Vázquez, D., Sánchez Hernández, M.I. y Corchuelo Martínez-Azúa, M.B. (2013). Validación de un instrumento de medida para la relación entre la orientación a la Responsabilidad Social Corporativa y otras variables estratégicas de la empresa. Revista de Contabilidad. Spanish Accounting Review, 6 (1), 11 - 23.

Gallardo-Vazquez, D. y Sanchez-Hernandez, I. (2012). Information on corporate social responsibility and SME's environmental responsiveness: A regional study. Economics and Sociology, 5 (2), 103 - 115.

Ganapathy, S. P., Natarajan, J., Gunasekaran, A., y Subramanian, N. (2014). Influence of eco-innovation on indian manufacturing sector sustainable performance. International Journal of Sustainable Development and World Ecology, 21 (3), 198 - 209.

Glover, J.L., Champion, D., Daniels, K.J. y Dainty, A.J.D. (2014). An Institutional Theory perspective on sustainable practices acrross the supply chain. Sustainable Food Supply Chain Management, 152, 102-111.

ISO (2008). UNE-EN ISO 9001:2008. Sistemas de gestión de calidad. Requistos., ISO, Geneva.

Kaiser, H.F. (1970). A second generation Little Jiffy. Psychometrika, 35, 401 415.

Kostova, T. y Roth, K. (2002). Adoption o fan organizational practice by subsidiaries of multinational corporations: Institutional and relational effects. Academy of Management Journal, 45, 215 - 243.

REDMARKA UIMA-Universidad de A Coruña - CIECID

Año VII, Número 13, (2014), v I pp. 19-42

http://www.redmarka.org/

ISSN 1852-2300 
Kumar, N., Stern, L. y Anderson, J. (1993). Conducting interorganizational research using key informants. Academy of Management Journal, 36, 1633 1651.

Llamas, R. (2005). Un análisis institucional de la implantación de la Agenda Local 21 por los Ayuntamientos españoles. Tesis Doctoral, Universidad de Granada. Facultad de Ciencias Económicas y Empresariales.

Llamas-Sanchez, R., Garcia-Morales, V. y Martin-Tapia, I. (2013). Factors affecting institutional change: A study of the adoption of Local Agenda 21 in Spain. Journal of Organizational Change Management, 26 (6), 1045 - 1070.

Meyer, J. y Rowan, B. (1977). Institutionalized organizations: Formal structure as mythand ceremony. The American Journal of Sociology, 83(2), 340-363.

Nunnally, J.C. (1978). Psychometric theory (2 ed.). New York: McGraw-Hill.

Pérez López, C. (2005). Métodos estadísticos avanzados con Spss. Madrid: Thomson.

Riquel Ligero, F.J. (2010), Análisis institucional de las prácticas de gestión ambiental de los campos de golf andaluces, Tesis Doctoral, Universidad de Huelva, España.

Rivera, J. (2004). Institutional pressures and voluntary environmental behavior in developing countries: Evidence from the Costa Rican hotel industry. Society and Natural Resources, 17 (9), 779 - 797.

Sánchez-Fernández, M.D. (2014). La Teoría Institucional y la Responsabilidad Social Corporativa en el sector hotelero de la Eurorregión Galicia-Norte de Portugal. Tesis Doctoral, Universidade da Coruña, Facultad de Económicas y Empresariales, España.

Sánchez-Fernández, M.D., Vargas-Sánchez, A. y Remoaldo, P. (2014). Institutional Context and Hotel Social Responsibility. Kybernetes, 43(3/4), 413-426.

Scott, W.R. (1995). Institutions and organizations. Thousand Oaks: Sage.

Sila, I. (2007). Examining the effects of contextual factors on TQM and performance through the lens of organizational theories: an empirical study. Journal of Operations Management, 25, 83 - 109.

Singh, J.V.; Tucker, D.J. y House, R.J. (1986). Organizational legitimacy and the liability of newness. Administrative Science Quarterly, 31, 171-193.

Suchman, M. (1995). Managing legitimacy: Strategic and institutional approaches. Academy of Management Review, 20 (3), 571 - 610.

Vanhonacker, W. (2000). A better way to crack China. Harvard Business Review, 78(4), 20-22.

Vargas-Sánchez, A. y Riquel-Ligero, F. (2012). Influence of the institutional context on the performance of golf courses, considering the natural

REDMARKA UIMA-Universidad de A Coruña - CIECID

Año VII, Número 13, (2014), v I pp. 19-42

http://www.redmarka.org/

ISSN 1852-2300 
environment. Environmental Engineering and Management Journal, 11 (11), 2001 - 2012.

Verdu Jover, A.J. (2002). Relación entre flexibilidad y desempeño organizativo: una aproximación desde la perspectiva de la gestión de la calidad total. Alicante: Universidad Miguel Hernández.

Wang, C.H. (2005). Constructing multivariate process capability indices for short-run production. Int.J. Adv. Manuf. Technol, 26, 1306 - 1311.

Wubneh, M.A. (1987). Multivariate analysis of socio-economic charasteristics of urban areas in Ethiopia. Afr. Urban Quaterly, 2, 425 - 433.

REDMARKA UIMA-Universidad de A Coruña - CIECID

Año VII, Número 13, (2014), v I pp. 19-42

http://www.redmarka.org/

ISSN 1852-2300 